\title{
Renormalisation of composite operators in lattice perturbation theory with clover fermions: non-forward matrix elements
}

\author{
M. Göckeler ${ }^{1, a}$, R. Horsley ${ }^{2}$, H. Perlt ${ }^{3}$, P.E.L. Rakow ${ }^{4}$, A. Schäfer ${ }^{1}$, G. Schierholz ${ }^{5,6}$, A. Schiller ${ }^{3}$ \\ ${ }^{1}$ Institut für Theoretische Physik, Universität Regensburg, 93040 Regensburg, Germany \\ 2 School of Physics, University of Edinburgh, Edinburgh EH9 3JZ, UK \\ 3 Institut für Theoretische Physik, Universität Leipzig, 04109 Leipzig, Germany \\ 4 Theoretical Physics Division, Department of Mathematical Sciences, University of Liverpool, Liverpool L69 3BX, UK \\ 5 John von Neumann-Institut für Computing NIC, Deutsches Elektronen-Synchrotron DESY, 15738 Zeuthen, Germany \\ ${ }^{6}$ Deutsches Elektronen-Synchrotron DESY, 22603 Hamburg, Germany
}

Received: 7 June 2006 /

Published online: 24 October 2006 - C S Springer-Verlag / Società Italiana di Fisica 2006

\begin{abstract}
We consider the renormalisation of lattice QCD operators with one and two covariant derivatives related to the first and second moments of generalised parton distributions and meson distribution amplitudes. Employing the clover fermion action we calculate their non-forward quark matrix elements in one-loop lattice perturbation theory. For some representations of the hypercubic group commonly used in simulations we determine the sets of all possible mixing operators and compute the matrices of the renormalisation factors in one-loop approximation. We describe how tadpole improvement is applied to the results.
\end{abstract}

PACS. 11.15.Ha; 12.38.Bx; 12.38.Gc

\section{Introduction}

Many interesting observables in hadron physics, e.g. (moments of) generalised parton distributions (GPDs) [1-5] or distribution amplitudes, can be computed from matrix elements of local operators between hadron states. (For an extensive review of GPDs see [6], for distribution amplitudes see, e.g., $[7,8]$.) GPDs, in particular, have attracted a lot of interest in recent years. They parametrise a large class of hadronic correlators, including e.g. form factors and the ordinary parton distribution functions. Thus GPDs provide a formal framework to connect information from various inclusive, semi-inclusive and exclusive reactions. Furthermore they give access to physical quantities which cannot be directly determined in experiments, like e.g. the orbital angular momentum of quarks and gluons in a nucleon (in a given scheme) and the spatial distribution of the energy or spin density of a fast moving hadron in the transverse plane. On the other hand, direct experimental information is limited and additional input is required to obtain a more complete knowledge of GPDs. One important source is lattice QCD, which can provide the relevant hadronic matrix elements [9-13].

Compared with moments of ordinary parton distributions, the specific difficulty in the treatment of moments of

\footnotetext{
a e-mail: meinulf.goeckeler@physik.uni-regensburg.de
}

GPDs and distribution amplitudes lies in the fact that the required matrix elements of local operators are no longer forward matrix elements. In general, this circumstance complicates the pattern of mixing under renormalisation, and more extended investigations become necessary.

In a recent publication [14] we have calculated the nonforward quark matrix elements needed for the renormalisation of quark-antiquark operators with two derivatives, which determine the second moments of GPDs, and we have discussed the mixing problem in detail. This calculation was performed in one-loop lattice perturbation theory for the Wilson fermion action.

In the present paper we extend our perturbative calculations to improved fermions using the SheikholeslamiWohlert (clover) action [15] for improving the vertices. We calculate renormalisation factors, but no improvement coefficients. While the general framework is of course the same as for Wilson fermions, the additional clover term in the action leads to a considerable complication of the calculations. A preliminary account of our work has already been given in [16]. Note, however, that a few misprints in [16] will be corrected here.

Let us fix the notation used in our perturbative calculations. We work in Euclidean space and employ the Wilson gauge action together with clover fermions such that the total action is given by

$$
S^{\text {latt }}=S_{\mathrm{SW}, \mathrm{F}}+S_{\mathrm{W}, \mathrm{G}} .
$$


The fermionic part $S_{\mathrm{SW}, \mathrm{F}}$ has the form [15]

$$
\begin{aligned}
S_{\mathrm{SW}, \mathrm{F}}= & 4 r a^{3} \sum_{x} \bar{\psi}(x) \psi(x) \\
& -\frac{a^{3}}{2} \sum_{x, \mu}\left[\bar{\psi}(x)\left(r-\gamma_{\mu}\right) U_{x, \mu} \psi(x+a \hat{\mu})\right. \\
& \left.+\bar{\psi}(x+a \hat{\mu})\left(r+\gamma_{\mu}\right) U_{x, \mu}^{\dagger} \psi(x)\right] \\
& -\mathrm{i} \frac{a^{5} g c_{\mathrm{Sw}}}{8} \sum_{x, \mu, \nu} \bar{\psi}(x)\left[\gamma_{\mu}, \gamma_{\nu}\right] F_{\mu \nu}^{\text {clover }} \psi(x),
\end{aligned}
$$

written in terms of dimensionful massless fermion fields $\psi(x)$. Here $a$ denotes the lattice spacing and the sums run over all lattice sites $x$ and directions $\mu, \nu$. All other indices are suppressed. $F_{\mu \nu}^{\text {clover }}$ is the standard "clover-leaf" form of the lattice field strength (see, e.g., Appendix D in [17]). The coupling strength of the improvement term is given by $c_{\mathrm{sw}}$. The link matrices $U_{x, \mu}$ are related to the gauge field $A_{\mu}(x)$ by

$$
U_{x, \mu}=\exp \left[\mathrm{iga} A_{\mu}(x)\right], \quad A_{\mu}(x)=T^{c} A_{\mu}^{c}(x),
$$

where $g$ is the bare gauge coupling and the $T^{c}$ are the generators of the $S U(3)$ algebra. The gauge action for the gluon field $A_{\mu}(x)$ is

$$
S_{\mathrm{W}, \mathrm{G}}=\frac{6}{g^{2}} \sum_{x, \mu<\nu}\left[1-\frac{1}{6} \operatorname{Tr}\left(U_{x, \mu \nu}+U_{x, \mu \nu}^{\dagger}\right)\right],
$$

with

$$
U_{x, \mu \nu}=U_{x, \mu} U_{x+a \hat{\mu}, \nu} U_{x+a \hat{\nu}, \mu}^{\dagger} U_{x, \nu}^{\dagger} .
$$

In a perturbative calculation the investigated operators are considered between off-shell quark states. Our calculations are performed in Feynman gauge; the final numbers will be presented for the Wilson parameter $r=1$, but for arbitrary values of $c_{\mathrm{sw}}$.

On the lattice the operators are classified according to the irreducible representations $\tau_{k}^{(l)}$ of the hypercubic group $H(4)$ (for the notation see, e.g., [18]). Here $l$ denotes the dimension of the representation and $k$ labels inequivalent representations of the same dimension. In addition our operators will be chosen such that they have definite charge conjugation parity $C$.

Using clover fermions, quark-antiquark operators with one covariant derivative have been discussed in [19] for forward matrix elements. Because of the constraints imposed by charge conjugation invariance no additional mixing has to be considered in non-forward matrix elements and the renormalisation constants given there can be taken over to the case at hand. We will present them again for completeness and add the corresponding results for operators involving $\left[\gamma_{\mu}, \gamma_{\nu}\right]$, which were not considered in [19].

In [14] the renormalisation procedure for the case of non-vanishing momentum transfer has been discussed in detail. We will not repeat this discussion here. The matrix of renormalisation and mixing coefficients $Z_{i j}(a \mu)$ relating regularised lattice vertex functions $\Gamma_{j}^{\mathrm{L}}\left(p^{\prime}, p, a, g_{\mathrm{R}}\right)$ and
$\overline{\mathrm{MS}}$ renormalised vertex functions $\Gamma_{i}^{\mathrm{R}}\left(p^{\prime}, p, \mu, g_{\mathrm{R}}\right)$ is defined such that

$$
\Gamma_{i}^{\mathrm{R}}\left(p^{\prime}, p, \mu, g_{\mathrm{R}}\right)=Z_{\psi}^{-1}(a \mu) \sum_{j=1}^{N} Z_{i j}(a \mu) \Gamma_{j}^{\mathrm{L}}\left(p^{\prime}, p, a, g_{\mathrm{R}}\right),
$$

with the quark wave function renormalisation constant $Z_{\psi}$. Here $p\left(p^{\prime}\right)$ denotes the momentum of the incoming (outgoing) quark, the renormalisation scale is $\mu$, the renormalised coupling is denoted by $g_{\mathrm{R}}$, and $N$ is the number of operators which mix in the one-loop approximation.

\section{Operators and mixing}

We consider operators with up to two covariant symmetric lattice derivatives $\stackrel{\leftrightarrow}{D}=\vec{D}-\overleftarrow{D}$ and external ordinary derivatives $\partial$. In the operator symbols, the derivatives are indicated by superscripts $D$ and $\partial$. Note that matrix elements of operators which are ordinary derivatives of other operators are simply given by the matrix elements of these other operators multiplied by the appropriate product of components of the momentum transfer.

The quark-antiquark operators with one derivative are given by

$$
\begin{aligned}
\mathcal{O}_{\mu \nu}^{D} & =-\frac{\mathrm{i}}{2} \bar{\psi} \gamma_{\mu} \stackrel{\leftrightarrow}{D}_{\nu} \psi, \\
\mathcal{O}_{\mu \nu}^{5, D} & =-\frac{\mathrm{i}}{2} \bar{\psi} \gamma_{\mu} \gamma_{5} \stackrel{\leftrightarrow}{D}_{\nu} \psi, \\
\mathcal{O}_{\mu \nu \omega}^{T, D} & =-\frac{\mathrm{i}}{2} \bar{\psi}\left[\gamma_{\mu}, \gamma_{\nu} \stackrel{\leftrightarrow}{D}_{\omega} \psi,\right. \\
\mathcal{O}_{\mu \nu \omega}^{T, \partial} & =-\frac{\mathrm{i}}{2} \partial_{\omega}\left(\bar{\psi}\left[\gamma_{\mu}, \gamma_{\nu}\right] \psi\right)
\end{aligned}
$$

Operators such as (9) involving $\left[\gamma_{\mu}, \gamma_{\nu}\right]$ are of interest for tensor GPDs as well as for transversity and we call them transversity operators. They are antisymmetric in the indices $\mu$ and $\nu$. For non-chiral fermions, operators (9) and (10) contribute as lower-dimensional operators to mixing in certain operators which determine second moments of GPDs.

As operators with two derivatives we consider

$$
\begin{aligned}
\mathcal{O}_{\mu \nu \omega}^{D D} & =-\frac{1}{4} \bar{\psi} \gamma_{\mu} \stackrel{\leftrightarrow}{D}_{\nu} \stackrel{\leftrightarrow}{D}_{\omega} \psi \\
\mathcal{O}_{\mu \nu \omega}^{\partial D} & =-\frac{1}{4} \partial_{\nu}\left(\bar{\psi} \gamma_{\mu} \stackrel{\leftrightarrow}{D}_{\omega} \psi\right) \\
\mathcal{O}_{\mu \nu \omega}^{\partial \partial} & =-\frac{1}{4} \partial_{\nu} \partial_{\omega}\left(\bar{\psi} \gamma_{\mu} \psi\right)
\end{aligned}
$$

and

$$
\begin{aligned}
\mathcal{O}_{\mu \nu \omega}^{5, D D} & =-\frac{1}{4} \bar{\psi} \gamma_{\mu} \gamma_{5} \stackrel{\leftrightarrow}{D}_{\nu} \stackrel{\leftrightarrow}{D}_{\omega} \psi \\
\mathcal{O}_{\mu \nu \omega}^{5, \partial D} & =-\frac{1}{4} \partial_{\nu}\left(\bar{\psi} \gamma_{\mu} \gamma_{5} \stackrel{\leftrightarrow}{D}_{\omega} \psi\right) \\
\mathcal{O}_{\mu \nu \omega}^{5, \partial \partial} & =-\frac{1}{4} \partial_{\nu} \partial_{\omega}\left(\bar{\psi} \gamma_{\mu} \gamma_{5} \psi\right)
\end{aligned}
$$


We include here also transversity operators with two derivatives:

$$
\begin{aligned}
\mathcal{O}_{\mu \nu \omega \sigma}^{T, D D} & =-\frac{1}{4} \bar{\psi}\left[\gamma_{\mu}, \gamma_{\nu}\right] \stackrel{\leftrightarrow}{D}_{\omega} \stackrel{\leftrightarrow}{D}_{\sigma} \psi, \\
\mathcal{O}_{\mu \nu \omega \sigma}^{T, \partial \partial \partial} & =-\frac{1}{4} \partial_{\omega} \partial_{\sigma}\left(\bar{\psi}\left[\gamma_{\mu}, \gamma_{\nu}\right] \psi\right)
\end{aligned}
$$

A detailed description of the operators with two derivatives, their representations and potential mixings is given in [14]. To define the various operators we use the following short-hand notation:

$$
\begin{aligned}
\mathcal{O}_{\ldots\left\{\nu_{1} \nu_{2}\right\}}= & \frac{1}{2}\left(\mathcal{O}_{\ldots \nu_{1} \nu_{2}}+\mathcal{O}_{\ldots \nu_{2} \nu_{1}}\right) \\
\mathcal{O}_{\left\{\nu_{1} \nu_{2} \nu_{3}\right\}}= & \frac{1}{6}\left(\mathcal{O}_{\nu_{1} \nu_{2} \nu_{3}}+\mathcal{O}_{\nu_{1} \nu_{3} \nu_{2}}+\mathcal{O}_{\nu_{2} \nu_{1} \nu_{3}}+\mathcal{O}_{\nu_{2} \nu_{3} \nu_{1}}\right. \\
& \left.+\mathcal{O}_{\nu_{3} \nu_{1} \nu_{2}}+\mathcal{O}_{\nu_{3} \nu_{2} \nu_{1}}\right) \\
\mathcal{O}_{\left\|\nu_{1} \nu_{2} \nu_{3}\right\|}= & \mathcal{O}_{\nu_{1} \nu_{2} \nu_{3}}-\mathcal{O}_{\nu_{1} \nu_{3} \nu_{2}}+\mathcal{O}_{\nu_{3} \nu_{1} \nu_{2}}-\mathcal{O}_{\nu_{3} \nu_{2} \nu_{1}} \\
& -2 \mathcal{O}_{\nu_{2} \nu_{3} \nu_{1}}+2 \mathcal{O}_{\nu_{2} \nu_{1} \nu_{3}} \\
\mathcal{O}_{\left\langle\left\langle\nu_{1} \nu_{2} \nu_{3}\right\rangle\right\rangle}= & \mathcal{O}_{\nu_{1} \nu_{2} \nu_{3}}+\mathcal{O}_{\nu_{1} \nu_{3} \nu_{2}}-\mathcal{O}_{\nu_{3} \nu_{1} \nu_{2}}-\mathcal{O}_{\nu_{3} \nu_{2} \nu_{1}}
\end{aligned}
$$

For the first moments we choose the representations and operators (for a more detailed discussion of the transformation under $H(4)$ see [18]) as in Table 1.

All operators in Table 1 are multiplicatively renormalisable. These representations exhaust all possibilities for the twist-2 sector in the continuum. Note that in [16] we have erroneously assigned $\tau_{1}^{(8)}$ and $C=-1$ to an operator belonging to $\tau_{1}^{(8)}, C=+1$.

Let us now turn to the second moments and the corresponding twist-2 operators.

First, in the unpolarised case we consider the following sets of mixing operators.

$\tau_{2}^{(4)}, C=-1$ :

We have

$$
\mathcal{O}_{\{124\}}^{D D}, \mathcal{O}_{\{124\}}^{\partial \partial}
$$

Table 1. Representations and operators chosen for the first moments

\begin{tabular}{lcc}
\hline Operator & Representation & $C$ \\
\hline $\mathcal{O}_{\{14\}}^{D}$ & $\tau_{3}^{(6)}$ & +1 \\
$\mathcal{O}_{44}^{D}-\frac{1}{3}\left(\mathcal{O}_{11}^{D}+\mathcal{O}_{22}^{D}+\mathcal{O}_{33}^{D}\right)$ & $\tau_{1}^{(3)}$ & +1 \\
$\mathcal{O}_{\{14\}}^{5, D}$ & $\tau_{4}^{(6)}$ & -1 \\
$\mathcal{O}_{44}^{5, D}-\frac{1}{3}\left(\mathcal{O}_{11}^{5, D}+\mathcal{O}_{22}^{5, D}+\mathcal{O}_{33}^{5, D}\right)$ & $\tau_{4}^{(3)}$ & -1 \\
$\mathcal{O}_{\langle\langle 124\rangle\rangle}^{T, D}$ & $\tau_{2}^{(8)}$ & +1 \\
$\mathcal{O}_{\langle\langle 122\rangle\rangle}^{T, D}-\mathcal{O}_{\langle\langle 133\rangle\rangle}^{T, D}$ & $\tau_{1}^{(8)}$ & +1 \\
\hline
\end{tabular}

$\tau_{1}^{(8)}, C=-1:$

Now we have

$$
\begin{aligned}
\mathcal{O}_{1} & =\mathcal{O}_{\{114\}}^{D D}-\frac{1}{2}\left(\mathcal{O}_{\{224\}}^{D D}+\mathcal{O}_{\{334\}}^{D D}\right), \\
\mathcal{O}_{2} & =\mathcal{O}_{\{114\}}^{\partial \partial}-\frac{1}{2}\left(\mathcal{O}_{\{224\}}^{\partial \partial}+\mathcal{O}_{\{334\}}^{\partial \partial}\right), \\
\mathcal{O}_{3} & =\mathcal{O}_{\langle\langle 114\rangle\rangle}^{D D}-\frac{1}{2}\left(\mathcal{O}_{\langle\langle 224\rangle\rangle}^{D D}+\mathcal{O}_{\langle\langle 334\rangle\rangle}^{D D}\right), \\
\mathcal{O}_{4} & =\mathcal{O}_{\langle\langle 114\rangle\rangle}^{\partial \partial}-\frac{1}{2}\left(\mathcal{O}_{\langle\langle 224\rangle\rangle}^{\partial \partial}+\mathcal{O}_{\langle\langle 334\rangle\rangle}^{\partial \partial}\right), \\
\mathcal{O}_{5} & =\mathcal{O}_{|| 213 \| \mid}^{5, \partial D}, \\
\mathcal{O}_{6} & =\mathcal{O}_{\langle\langle 213\rangle\rangle}^{5, \partial D}, \\
\mathcal{O}_{7} & =\mathcal{O}_{\|\mid 213\|}^{5, D D}, \\
\mathcal{O}_{8} & =\mathcal{O}_{411}^{T, \partial}-\frac{1}{2}\left(\mathcal{O}_{422}^{T, \partial}+\mathcal{O}_{433}^{T, \partial}\right) .
\end{aligned}
$$

There is one more representation, $\tau_{1}^{(4)}, C=-1$, giving twist-2 operators. However, even in forward matrix elements the corresponding operators mix with operators whose dimension is smaller by two. Therefore they are rather unsuitable for numerical simulations and will not be discussed any further.

In the polarised case we consider the following.

$\tau_{3}^{(4)}, C=+1:$

We have

$$
\mathcal{O}_{\{124\}}^{5, D D}, \mathcal{O}_{\{124\}}^{5, \partial \partial}
$$

$\tau_{2}^{(8)}, C=+1:$

We have

$$
\begin{aligned}
\mathcal{O}_{1}^{5} & =\mathcal{O}_{\{114\}}^{5, D D}-\frac{1}{2}\left(\mathcal{O}_{\{224\}}^{5, D D}+\mathcal{O}_{\{334\}}^{5, D D}\right), \\
\mathcal{O}_{2}^{5} & =\mathcal{O}_{\{114\}}^{5, \partial \partial}-\frac{1}{2}\left(\mathcal{O}_{\{224\}}^{5, \partial \partial}+\mathcal{O}_{\{334\}}^{5, \partial \partial}\right), \\
\mathcal{O}_{3}^{5} & =\mathcal{O}_{\langle\langle 114\rangle\rangle}^{5, D D}-\frac{1}{2}\left(\mathcal{O}_{\langle\langle 224\rangle\rangle}^{5, D D}+\mathcal{O}_{\langle\langle 334\rangle\rangle}^{5, D D}\right), \\
\mathcal{O}_{4}^{5} & =\mathcal{O}_{\langle\langle 114\rangle\rangle}^{5, \partial \partial}-\frac{1}{2}\left(\mathcal{O}_{\langle\langle 224\rangle\rangle}^{5, \partial \partial}+\mathcal{O}_{\langle\langle 334\rangle\rangle}^{5, \partial \partial}\right), \\
\mathcal{O}_{5}^{5} & =\mathcal{O}_{|| 213||}^{\partial D}, \\
\mathcal{O}_{6}^{5} & =\mathcal{O}_{\langle\langle 213\rangle\rangle}^{\partial D}, \\
\mathcal{O}_{7}^{5} & =\mathcal{O}_{|| 213 \|}^{D D}, \\
\mathcal{O}_{8}^{5} & =\mathcal{O}_{123}^{T, D}-2 \mathcal{O}_{231}^{T, D}-\mathcal{O}_{132}^{T, D} .
\end{aligned}
$$

Again, there is one more representation giving twist-2 operators. Here it is $\tau_{4}^{(4)}, C=+1$, but the corresponding operators suffer from similar mixing problems as the operators with $\tau_{1}^{(4)}, C=-1$ in the unpolarised case and will be omitted in the following. 
$\tau_{2}^{(3)}, C=-1:$

Here

$$
\mathcal{O}_{1}^{T}=\mathcal{O}_{4\{123\}}^{T, D D}, \mathcal{O}_{2}^{T}=\mathcal{O}_{4\{123\}}^{T, \partial \partial}
$$

$\tau_{3}^{(3)}, C=-1:$

In this case

$\mathcal{O}_{3}^{T}=-\mathcal{O}_{1\{133\}}^{T, D D}+\mathcal{O}_{1\{144\}}^{T, D D}-\mathcal{O}_{2\{233\}}^{T, D D}+\mathcal{O}_{2\{244\}}^{T, D D}-2 \mathcal{O}_{3\{344\}}^{T, D D}$
$\mathcal{O}_{4}^{T}=-\mathcal{O}_{1\{133\}}^{T, \partial \partial}+\mathcal{O}_{1\{144\}}^{T, \partial \partial}-\mathcal{O}_{2\{233\}}^{T, \partial \partial}+\mathcal{O}_{2\{244\}}^{T, \partial \partial}-2 \mathcal{O}_{3\{344\}}^{T, \partial \partial}$

$\tau_{2}^{(6)}, C=-1:$

We have

$$
\begin{aligned}
& \mathcal{O}_{5}^{T}=\mathcal{O}_{13\{32\}}^{T, D D}+\mathcal{O}_{23\{31\}}^{T, D D}-\mathcal{O}_{14\{42\}}^{T, D D}-\mathcal{O}_{24\{41\}}^{T, D D} \\
& \mathcal{O}_{6}^{T}=\mathcal{O}_{13\{32\}}^{T, \partial \partial}+\mathcal{O}_{23\{31\}}^{T, \partial \partial}-\mathcal{O}_{14\{42\}}^{T, \partial \partial}-\mathcal{O}_{24\{41\}}^{T, \partial \partial}
\end{aligned}
$$

The representations (22), (23) and (24) exhaust all possibilities for transversity operators of twist 2 which have a mixing matrix of size $2 \times 2$ only. Other representations have more complicated mixing patterns.

\section{One-loop calculation}

We calculate the matrix elements of the operators in oneloop lattice perturbation theory in the infinite volume limit following Kawai et al. [20]. Details of the computational procedure, in particular the Feynman rules, are given in [14].

\subsection{First moment}

Since mixing is absent we need no matrix indices for the renormalisation constants and use the general form

$$
Z(a \mu)=1-\frac{g_{\mathrm{R}}^{2} C_{\mathrm{F}}}{16 \pi^{2}}\left(\gamma \ln \left(a^{2} \mu^{2}\right)+B\left(c_{\mathrm{sw}}\right)\right)
$$

where the finite piece $B$ depends on $c_{\mathrm{sw}}$ and the (one-loop) anomalous dimension $\gamma$ is given by

$$
\gamma= \begin{cases}8 / 3 & \text { for } \tau_{3}^{(6)}, \tau_{1}^{(3)}, \tau_{4}^{(6)}, \tau_{4}^{(3)} \\ 2 & \text { for } \tau_{2}^{(8)}, \tau_{1}^{(8)}\end{cases}
$$

For the operators in Table 1 we have (results for $\tau_{3}^{(6)}, \tau_{1}^{(3)}$, $\tau_{4}^{(6)}$ and $\tau_{4}^{(3)}$ are taken from [19]) the expressions of Table 2.
Table 2. Values of $B\left(c_{\mathrm{Sw}}\right)$ for representations of Table 1

\begin{tabular}{lc}
\hline Representation & $B\left(c_{\mathrm{Sw}}\right)$ \\
\hline$\tau_{3}^{(6)}$ & $1.27959-3.87297 c_{\mathrm{Sw}}-0.67826 c_{\mathrm{Sw}}^{2}$ \\
$\tau_{1}^{(3)}$ & $2.56184-3.96980 c_{\mathrm{Sw}}-1.03973 c_{\mathrm{Sw}}^{2}$ \\
$\tau_{4}^{(6)}$ & $0.34512-1.35931 c_{\mathrm{Sw}}-1.89255 c_{\mathrm{Sw}}^{2}$ \\
$\tau_{4}^{(3)}$ & $0.16738-1.24953 c_{\mathrm{Sw}}-1.99804 c_{\mathrm{Sw}}^{2}$ \\
$\tau_{2}^{(8)}$ & $13.16895+2.67533 c_{\mathrm{Sw}}-1.49375 c_{\mathrm{Sw}}^{2}$ \\
$\tau_{1}^{(8)}$ & $12.80396+2.62368 c_{\mathrm{Sw}}-1.42975 c_{\mathrm{Sw}}^{2}$ \\
\hline
\end{tabular}

\subsection{Second moment}

We write the matrix of renormalisation constants in the generic form

$$
Z_{i j}^{(m)}(a \mu)=\delta_{i j}-\frac{g_{\mathrm{R}}^{2} C_{\mathrm{F}}}{16 \pi^{2}}\left(\gamma_{i j} \ln \left(a^{2} \mu^{2}\right)+B_{i j}^{(m)}\left(c_{\mathrm{sw}}\right)\right),
$$

with $^{1}$

$$
B_{i j}^{(m)}\left(c_{\mathrm{sw}}\right)=B_{i j}^{(0, m)}+B_{i j}^{(1, m)} c_{\mathrm{sw}}+B_{i j}^{(2, m)} c_{\mathrm{sw}}^{2} .
$$

The superscript $(m)$ with $m=$ I, II distinguishes the realisations I and II of the covariant derivatives, which are explained in Appendix A of [14]. In the first case the momentum transfer "acts" at the position $x$ associated with the operator, where we define for one covariant derivative

$$
\begin{aligned}
\mathcal{O}(x)= & \left(\bar{\psi} \stackrel{\leftrightarrow}{D}_{\mu} \psi\right)(x) \\
= & \frac{1}{2 a}\left(\bar{\psi}(x) U_{x, \mu} \psi(x+a \hat{\mu})-\bar{\psi}(x) U_{x-a \hat{\mu}, \mu}^{\dagger} \psi(x-a \hat{\mu})\right. \\
& \left.+\bar{\psi}(x-a \hat{\mu}) U_{x-a \hat{\mu}, \mu} \psi(x)-\bar{\psi}(x+a \hat{\mu}) U_{x, \mu}^{\dagger} \psi(x)\right) .
\end{aligned}
$$

We have set the Dirac matrix in the operator equal to the unit matrix for simplicity. Realisation I leads to

$$
\begin{aligned}
\left(\bar{\psi} \stackrel{\leftrightarrow}{D}_{\mu} \psi\right)^{(\mathrm{I})}(q)= & \frac{1}{2 a} \sum_{x}\left[\bar{\psi}(x) U_{x, \mu} \psi(x+a \hat{\mu})\right. \\
& \left.-\bar{\psi}(x+a \hat{\mu}) U_{x, \mu}^{\dagger} \psi(x)\right]\left[\mathrm{e}^{\mathrm{i} q x}+\mathrm{e}^{\mathrm{i} q(x+a \hat{\mu})}\right]
\end{aligned}
$$

Alternatively (realisation II), $q$ can be applied at the point half way between the $\bar{\psi}$ and $\psi$ fields:

$$
\begin{aligned}
\left(\bar{\psi} \stackrel{\leftrightarrow}{D}_{\mu} \psi\right)^{(\mathrm{II})}(q)= & \frac{1}{a} \sum_{x}\left[\bar{\psi}(x) U_{x, \mu} \psi(x+a \hat{\mu})\right. \\
& \left.-\bar{\psi}(x+a \hat{\mu}) U_{x, \mu}^{\dagger} \psi(x)\right] \mathrm{e}^{\mathrm{i} q(x+a \hat{\mu} / 2)}
\end{aligned}
$$

${ }^{1} B_{i j}^{(0, m)}$ has been denoted by $c_{i j}^{(m)}$ in [14]. 
In the case of two covariant derivatives we have for realisation II

$$
\begin{aligned}
\left(\bar{\psi} \stackrel{\leftrightarrow}{D}_{\mu} \stackrel{\leftrightarrow}{D}_{\nu} \psi\right)^{(\mathrm{II})}(q) \\
=\frac{1}{a^{2}} \sum_{x}\left(\bar{\psi}(x) U_{x, \mu} U_{x+a \hat{\mu}, \nu} \psi(x+a \hat{\mu}+a \hat{\nu})\right. \\
\quad-\bar{\psi}(x+a \hat{\nu}) U_{x+a \hat{\nu}, \mu} U_{x+a \hat{\mu}, \nu}^{\dagger} \psi(x+a \hat{\mu}) \\
\quad-\bar{\psi}(x+a \hat{\mu}) U_{x, \mu}^{\dagger} U_{x, \nu} \psi(x+a \hat{\nu}) \\
\left.\quad+\bar{\psi}(x+a \hat{\mu}+a \hat{\nu}) U_{x+a \hat{\nu}, \mu}^{\dagger} U_{x, \nu}^{\dagger} \psi(x)\right) \mathrm{e}^{\mathrm{i} q(x+a \hat{\mu} / 2+a \hat{\nu} / 2)}
\end{aligned}
$$

and realisation I is obtained from (32) as

$$
\begin{aligned}
\left(\bar{\psi} \stackrel{\leftrightarrow}{D}_{\mu} \stackrel{\leftrightarrow}{D}_{\nu} \psi\right)^{(\mathrm{I})}(q)= & \cos \left(\frac{a q_{\mu}}{2}\right) \cos \left(\frac{a q_{\nu}}{2}\right) \\
& \times\left(\bar{\psi} \stackrel{\leftrightarrow}{D}_{\mu} \stackrel{\leftrightarrow}{D}_{\nu} \psi\right)^{(\mathrm{II})}(q)
\end{aligned}
$$

We get the following results.

$\mathcal{O}_{\{124\}}^{D D}\left(\tau_{2}^{(4)}, C=-1\right):$

In this case we have the mixing operators (18). The corresponding $2 \times 2$ mixing matrices are

$$
\begin{aligned}
\gamma & =\left(\begin{array}{cc}
\frac{25}{6} & -\frac{5}{6} \\
0 & 0
\end{array}\right), \\
B^{(0, \mathrm{I}, \mathrm{II})} & =\left(\begin{array}{cc}
-11.56318 & 0.02414 \\
0 & 20.61780
\end{array}\right), \\
B^{(1, \mathrm{I}, \mathrm{II})} & =\left(\begin{array}{cc}
2.89800 & -0.25529 \\
0 & 4.74556
\end{array}\right), \\
B^{(2, \mathrm{I}, \mathrm{II})} & =\left(\begin{array}{cc}
-0.98387 & -0.01557 \\
0 & -0.54317
\end{array}\right) .
\end{aligned}
$$

The matrix $B^{(\mathrm{I}, \mathrm{II})}$ shows a rather small coefficient for the mixing between the operators $\mathcal{O}_{\{124\}}^{D D}$ and $\mathcal{O}_{\{124\}}^{\partial \partial}$. Thus it may be justified to neglect the mixing in practical applications, where $\mu=1 / a$.

$\mathcal{O}_{1}\left(\tau_{1}^{(8)}, C=-1\right):$

The operators mixing with $\mathcal{O}_{1}$ are given in (19). First we consider the operators of the same dimension $\mathcal{O}_{1}, \ldots, \mathcal{O}_{7}$. To one-loop accuracy the operator $\mathcal{O}_{7}$ does not contribute, because its Born term vanishes, and we have to consider the following mixing set:

$$
\left\{\mathcal{O}_{1}, \mathcal{O}_{2}, \mathcal{O}_{3}, \mathcal{O}_{4}, \mathcal{O}_{5}, \mathcal{O}_{6}\right\} .
$$

The anomalous dimension matrix is

$$
\gamma=\left(\begin{array}{cccccc}
\frac{25}{6} & -\frac{5}{6} & 0 & 0 & 0 & 0 \\
0 & 0 & 0 & 0 & 0 & 0 \\
0 & 0 & \frac{7}{6} & -\frac{5}{6} & 1 & -\frac{3}{2} \\
0 & 0 & 0 & 0 & 0 & 0 \\
0 & 0 & 0 & 0 & 2 & -2 \\
0 & 0 & 0 & 0 & -\frac{2}{3} & \frac{2}{3}
\end{array}\right),
$$

and the finite parts of the mixing matrix are given by (in doublets the upper number belongs to type I, the lower to type II of the realisation of the lattice covariant derivative)

$B^{(0, \mathrm{I}, \mathrm{II})}$

$$
=\left(\begin{array}{cccccc}
-12.12740 & \left(\begin{array}{r}
1.49127 \\
-2.73669
\end{array}\right) & 0.36848 & \left(\begin{array}{r}
-0.41595 \\
0.99336
\end{array}\right) & 0.01562 & 0.14983 \\
0 & 20.61780 & 0 & 0 & 0 \\
3.30605 & \left(\begin{array}{c}
-8.01456 \\
18.18411
\end{array}\right) & -14.85157 & \left(\begin{array}{c}
4.43061 \\
-4.30228
\end{array}\right) & -0.92850 & 0.73802 \\
0 & 0 & 0 & 20.61780 & 0 & 0 \\
0 & 3.26440 & 0 & 0 & 0.35008 & 0.01491 \\
0 & 3.26440 & 0 & 0 & 0.00497 & 0.36003
\end{array}\right) \text {, }
$$

$B^{(1, \mathrm{I}, \mathrm{II})}$

$$
=\left(\begin{array}{cccccc}
2.92169 & \left(\begin{array}{l}
-0.21269 \\
-0.68643
\end{array}\right) & -0.03276 & \left(\begin{array}{l}
0.01492 \\
0.17283
\end{array}\right) & -0.01878 & 0.05696 \\
0 & 4.74556 & 0 & 0 & 0 & 0 \\
0.33335 & \left(\begin{array}{c}
-0.76570 \\
-0.05510
\end{array}\right) & 2.15228 & \left(\begin{array}{c}
1.20652 \\
0.96966
\end{array}\right) & -1.75814 & 2.29837 \\
0 & 0 & 0 & 4.74556 & 0 & 0 \\
0 & -1.44106 & 0 & 0 & 1.64790 & 0.86576 \\
0 & -1.44106 & 0 & 0 & 0.28859 & 2.22507
\end{array}\right),
$$

$B^{(2, \mathrm{I}, \mathrm{II})}$

$$
=\left(\begin{array}{cccccc}
-0.98166 & \left(\begin{array}{l}
-0.07815 \\
-0.10117
\end{array}\right) & -0.02914 & \left(\begin{array}{l}
0.03475 \\
0.04243
\end{array}\right) & -0.00999 & 0.00688 \\
0 & -0.54317 & 0 & 0 & 0 & 0 \\
0.37050 & \left(\begin{array}{c}
-0.55068 \\
0.21545
\end{array}\right) & -1.70741 & \left(\begin{array}{c}
0.37132 \\
0.11594
\end{array}\right) & -0.44295 & 0.10328 \\
0 & 0 & 0 & -0.54317 & 0 & 0 \\
0 & 1.41570 & 0 & 0 & -1.70334 & 0.56763 \\
0 & 1.41570 & 0 & 0 & 0.18921 & -1.32493
\end{array}\right) .
$$

The matrices $B^{(k, \mathrm{I}, \mathrm{II})}$ show sizeable coefficients for the mixing of $\mathcal{O}_{1}$ with other operators, especially with $\mathcal{O}_{2}$ containing two external ordinary derivatives.

There is also a possible mixing between $\mathcal{O}_{1}$ and the lower-dimensional operator $\mathcal{O}_{8}$ in (19). Indeed, we find in the one-loop approximation that the vertex function of $\mathcal{O}_{1}$ contains a term $\propto 1 / a$ :

$$
\begin{aligned}
\left.\mathcal{O}_{1}\right|_{\frac{1}{a}-\mathrm{part}}= & \frac{g_{\mathrm{R}}^{2} C_{\mathrm{F}}}{16 \pi^{2}}\left(-0.51771+0.08325 c_{\mathrm{sw}}-0.00983 c_{\mathrm{sw}}^{2}\right) \\
& \times \frac{1}{a} \mathcal{O}_{8}^{\text {Born }} .
\end{aligned}
$$

This mixing leads to a contribution which diverges like the inverse lattice spacing in the continuum limit. Thus the perturbative calculation of the mixing coefficient is not reliable and the operator $\mathcal{O}_{8}$ has to be subtracted nonperturbatively from the operator $\mathcal{O}_{1}$.

$\mathcal{O}_{\{124\}}^{5, D D}\left(\tau_{3}^{(4)}, C=+1\right):$

In this case we have to consider the operators in (20). The anomalous dimension matrix is given by (34), the finite 
contributions are collected in the matrices

$$
\begin{aligned}
B^{(0, \mathrm{I}, \mathrm{II})} & =\left(\begin{array}{cc}
-12.11715 & 0.16673 \\
0 & 15.79628
\end{array}\right), \\
B^{(1, \mathrm{I}, \mathrm{II})} & =\left(\begin{array}{cc}
1.51925 & -0.00505 \\
0 & -0.24783
\end{array}\right), \\
B^{(2, \mathrm{I}, \mathrm{II})} & =\left(\begin{array}{cc}
-1.71846 & 0.00711 \\
0 & -2.25137
\end{array}\right) .
\end{aligned}
$$

As in the case of the operator $\mathcal{O}_{\{124\}}^{D D}$ above, the mixing coefficient is rather small.

$\mathcal{O}_{1}^{5}\left(\tau_{2}^{(8)}, C=+1\right):$

First we discuss the mixing of operators of the same dimension in (21). The set of contributing operators is found to be

$$
\left\{\mathcal{O}_{1}^{5}, \mathcal{O}_{2}^{5}, \mathcal{O}_{3}^{5}, \mathcal{O}_{4}^{5}, \mathcal{O}_{5}^{5}, \mathcal{O}_{6}^{5}\right\}
$$

As in the case of the operator $\mathcal{O}_{1}$, one operator - here $\mathcal{O}_{7}^{5}-$ does not contribute to mixing in one-loop order. The finite contributions are

$$
\begin{aligned}
& B^{(0, \mathrm{I}, \mathrm{II})} \\
& =\left(\begin{array}{cccccc}
-12.86094 & \left(\begin{array}{r}
-2.06532 \\
1.48943
\end{array}\right) & 0.34900 & \left(\begin{array}{r}
0.85381 \\
-0.33110
\end{array}\right) & 0.05113 & 0.05942 \\
0 & 15.79628 & 0 & 0 & 0 & 0 \\
3.42196 & \left(\begin{array}{c}
15.82073 \\
-7.30020
\end{array}\right) & -15.35920 & \left(\begin{array}{r}
-5.16392 \\
2.54306
\end{array}\right) & 0.17014 & -0.94314 \\
0 & 0 & 0 & 15.79628 & 0 & 0 \\
0 & -8.91237 & 0 & 0 & 0.95969 & -0.95969 \\
0 & -8.91237 & 0 & 0 & -0.31990 & 0.31990
\end{array}\right),
\end{aligned}
$$

$B^{(1, \mathrm{I}, \mathrm{II})}$

$$
=\left(\begin{array}{cccccc}
1.49316 & \left(\begin{array}{r}
-0.03027 \\
0.17480
\end{array}\right) & -0.00750 & \left(\begin{array}{r}
0.03022 \\
-0.09858
\end{array}\right) & 0.01290 & -0.03622 \\
0 & -0.24783 & 0 & 0 & 0 & 0 \\
-0.09099 & \left(\begin{array}{c}
0.99704 \\
-1.07971
\end{array}\right) & 2.30129 & \left(\begin{array}{cccc}
-1.25511 \\
-0.56286
\end{array}\right) & 0.13420 & 0.48232 \\
0 & 0 & 0 & -0.24783 & 0 & 0 \\
0 & -13.26724 & 0 & 0 & 3.27954 & -1.78029 \\
0 & -13.26724 & 0 & 0 & -0.59343 & 2.09268
\end{array}\right) \text {, }
$$

$B^{(2, \mathrm{I}, \mathrm{II})}$

$$
=\left(\begin{array}{cccccc}
-1.68673 & \left(\begin{array}{l}
0.03501 \\
0.19704
\end{array}\right) & -0.00612 & \left(\begin{array}{l}
-0.01275 \\
-0.06677
\end{array}\right) & 0.01000 & -0.00692 \\
0 & -2.25137 & 0 & 0 & 0 & 0 \\
0.16581 & \left(\begin{array}{l}
0.28263 \\
0.13256
\end{array}\right) & -1.36546 & \left(\begin{array}{cccc}
-0.30614 \\
-0.25600
\end{array}\right) & 0.44299 & -0.10322 \\
0 & 0 & 0 & -2.25137 & 0 & 0 \\
0 & -1.41570 & 0 & 0 & -0.97445 & -0.88857 \\
0 & -1.41570 & 0 & 0 & -0.29619 & -1.56683
\end{array}\right) .
$$

The anomalous dimension matrix is the same as for the operators without $\gamma_{5}$; see (39). Again, some of the mixing coefficients are non-negligible. The mixing between $\mathcal{O}_{1}^{5}$ and $\mathcal{O}_{3}^{5}$ is present also in the forward case.

We also find mixing with a lower-dimensional operator, in this case it is the operator $\mathcal{O}_{8}^{5}$ in $(21)$. The corresponding contribution in the vertex function of $\mathcal{O}_{1}^{5}$ reads

$$
\begin{aligned}
\left.\mathcal{O}_{1}^{5}\right|_{\frac{1}{a}-\text { part }}= & -\frac{g_{\mathrm{R}}^{2} C_{\mathrm{F}}}{16 \pi^{2}}\left(0.25231+0.02507 c_{\mathrm{sw}}+0.01046 c_{\mathrm{sw}}^{2}\right) \\
& \times \frac{1}{a} \mathcal{O}_{8}^{5, \text { Born }} .
\end{aligned}
$$

$\mathcal{O}_{1}^{T}\left(\tau_{2}^{(3)}, C=-1\right):$

For the operators (22) the matrix of anomalous dimensions is given by

$$
\gamma=\left(\begin{array}{cc}
\frac{13}{3} & -\frac{2}{3} \\
0 & 1
\end{array}\right)
$$

and the finite contributions are

$$
\begin{aligned}
B^{(0, \mathrm{I}, \mathrm{II})} & =\left(\begin{array}{cc}
-11.54826 & 0.21894 \\
0 & 17.01808
\end{array}\right), \\
B^{(1, \mathrm{I}, \mathrm{II})} & =\left(\begin{array}{cc}
2.41077 & 0.05383 \\
0 & 3.91333
\end{array}\right), \\
B^{(2, \mathrm{I}, \mathrm{II})} & =\left(\begin{array}{cc}
-1.51175 & -0.00614 \\
0 & -1.97230
\end{array}\right) .
\end{aligned}
$$

$\mathcal{O}_{3}^{T}\left(\tau_{3}^{(3)}, C=-1\right):$

The operators (23) have the same anomalous dimension matrix, (52), as the previous case. For the finite pieces we obtain

$$
\begin{aligned}
B^{(0, \mathrm{I}, \mathrm{II})} & =\left(\begin{array}{cc}
-11.86877 & 0.27533 \\
0 & 17.01808
\end{array}\right), \\
B^{(1, \mathrm{I}, \mathrm{II})} & =\left(\begin{array}{cc}
2.30651 & 0.01831 \\
0 & 3.91333
\end{array}\right), \\
B^{(2, \mathrm{I}, \mathrm{II})} & =\left(\begin{array}{rr}
-1.34908 & 0.01726 \\
0 & -1.97230
\end{array}\right) .
\end{aligned}
$$

$\mathcal{O}_{5}^{T}\left(\tau_{2}^{(6)}, C=-1\right):$

We find for the case (24) the finite mixing contributions

$$
\begin{aligned}
B^{(0, \mathrm{I}, \mathrm{II})} & =\left(\begin{array}{cc}
-11.74773 & 0.23797 \\
0 & 17.01808
\end{array}\right), \\
B^{(1, \mathrm{I}, \mathrm{II})} & =\left(\begin{array}{cc}
2.36201 & 0.04490 \\
0 & 3.91333
\end{array}\right), \\
B^{(2, \mathrm{I}, \mathrm{II})} & =\left(\begin{array}{cc}
-1.45084 & 0.00898 \\
0 & -1.97230
\end{array}\right),
\end{aligned}
$$

with the anomalous dimension matrix (52). 


\section{Tadpole improvement}

It is well known that many results of (naive) lattice perturbation theory do not agree very well with their counterparts determined from Monte Carlo calculations. One main reason for these discrepancies is the appearance of gluon tadpoles, which are typical lattice artefacts. They turn the bare coupling $g$ into a poor expansion parameter. As a remedy the so-called tadpole (or mean field) improvement has been proposed [21], a rearrangement of the perturbative series making use of the variable $u_{0}$, the fourth root of the measured value of the plaquette,

$$
u_{0}=\left\langle\frac{1}{N_{c}} \operatorname{Tr} U_{\square}\right\rangle^{\frac{1}{4}} .
$$

Its value depends on the coupling $g^{2}=6 / \beta$ where it has been measured.

In case of mixing the tadpole improvement works as follows. Scaling the link variables $U_{\mu}$ with $u_{0}$

$$
U_{\mu}(x)=u_{0}\left(\frac{U_{\mu}(x)}{u_{0}}\right)=u_{0} \bar{U}_{\mu}(x)
$$

one finds an expression for the vertex function $\Gamma_{j}$ of an operator $\mathcal{O}_{j}$ containing $n_{j}$ covariant derivatives which is of the form

$$
\Gamma_{j}\left(U_{\mu}(x)\right)=u_{0}^{n_{j}} \Gamma_{j}\left(\bar{U}_{\mu}(x)\right) .
$$

$\Gamma_{j}\left(\bar{U}_{\mu}(x)\right)=u_{0}^{-n_{j}} \Gamma_{j}\left(U_{\mu}(x)\right)$ is expected to have a better converging perturbative expansion, which is obtained by inserting the expansions of $u_{0}^{-n_{j}}$ and $\Gamma_{j}\left(U_{\mu}(x)\right)$. To oneloop accuracy, $u_{0}$ is given by

$$
u_{0}=1-\frac{g^{2} C_{\mathrm{F}}}{16 \pi^{2}} \pi^{2}+O\left(g^{4}\right)
$$

Note that at the one-loop level we do not have to distinguish between $g$ and $g_{\mathrm{R}}$ in the perturbative expressions. The exponent of $u_{0}$ depends on $j$ because in general the mixing operators have different numbers of covariant derivatives (see, e.g., (18) or (19)). An external ordinary derivative $(\partial)$ does not provide a factor of $u_{0}$. Taking into account the mean field value for the wave function renormalisation constant for massless Wilson and clover fermions,

$$
Z_{\psi}^{\mathrm{MF}}=u_{0},
$$

the mean field $Z$ factor for each operator $\mathcal{O}_{j}$ contributing to the mixing reads

$$
Z_{j}^{\mathrm{MF}}=u_{0}^{1-n_{j}},
$$

and the tadpole improved matrix of renormalisation constants is given by

$$
\begin{aligned}
Z_{i j}^{\mathrm{TI}} & =Z_{i j} \frac{Z_{j}^{\mathrm{MF}}}{Z_{j}^{\mathrm{MF}, \text { pert }}} \\
& =u_{0}^{1-n_{j}}\left(1-\frac{g^{2} C_{\mathrm{F}}}{16 \pi^{2}}\left(n_{j}-1\right) \pi^{2}+O\left(g^{4}\right)\right) Z_{i j} .
\end{aligned}
$$

Additionally, we replace the parameters $g$ and $c_{\mathrm{sw}}$ by their "boosted" counterparts:

$$
g_{\mathrm{TI}}^{2} \equiv g^{2} u_{0}^{-4}, \quad c_{\mathrm{sw}}^{\mathrm{TI}} \equiv c_{\mathrm{sw}} u_{0}^{3} .
$$

Combining (27), (68) and (69) we obtain for the tadpole improved matrix in one-loop order

$$
\begin{aligned}
Z_{i j}^{\mathrm{TI},(m)}= & u_{0}^{1-n_{j}}\left(\delta_{i j}-\frac{g_{\mathrm{TI}}^{2} C_{\mathrm{F}}}{16 \pi^{2}}\left(\gamma_{i j} \ln \left(a^{2} \mu^{2}\right)\right.\right. \\
& \left.\left.+B_{i j}^{(m)}\left(c_{\mathrm{sw}}^{\mathrm{TI}}\right)+\left(n_{j}-1\right) \pi^{2} \delta_{i j}\right)\right) \\
\equiv & u_{0}^{1-n_{j}}\left(\delta_{i j}-\frac{g_{\mathrm{TI}}^{2} C_{\mathrm{F}}}{16 \pi^{2}}\left(\gamma_{i j} \ln \left(a^{2} \mu^{2}\right)\right.\right. \\
& \left.\left.+B_{i j}^{T I,(m)}\left(c_{\mathrm{sw}}^{\mathrm{TI}}\right)\right)\right) .
\end{aligned}
$$

Let us exemplify the impact of tadpole improvement in some typical cases. We choose $\mu=1 / a, c_{\mathrm{sw}}=1+O\left(g^{2}\right)$ and $u_{0}=0.8778$ corresponding to quenched calculations at $\beta=$ 6 . For operators with one covariant derivative the tadpole improvement procedure is rather simple. Because $n_{1}=1$ the only effect consists in replacing in (25) and Table $2 c_{\mathrm{sw}}$ by $c_{\mathrm{sw}}^{\mathrm{TI}}$ and $g$ by $g_{\mathrm{TI}}$. For the operators belonging to the representation $\tau_{3}^{(6)}$, e.g., we get

$$
Z=1.028 \quad \longrightarrow \quad Z^{\mathrm{TI}}=1.023 .
$$

The operators for the second moments of GPDs are a bit more involved. First we consider the simple mixing $\mathcal{O}_{\{124\}}^{5, D D} \leftrightarrow \mathcal{O}_{\{124\}}^{5, \partial 2} ;$ see $(20)$. Without tadpole improvement we obtain the mixing matrix of renormalisation constants as

$$
Z=\left(\begin{array}{cc}
1.10399 & -0.00143 \\
0 & 0.88773
\end{array}\right)
$$

The tadpole improved result is

$$
Z^{\mathrm{TI}}=\left(\begin{array}{cc}
1.17171 & -0.00208 \\
0 & 0.81876
\end{array}\right) .
$$

It is instructive to compare the one-loop corrections for the renormalisation constants, i.e. $B_{i j}^{(m)}\left(c_{\mathrm{sw}}\right)$ for the unimproved case $(27)$ and $B_{i j}^{\mathrm{TI},(m)}\left(c_{\mathrm{sw}}^{\mathrm{TI}}\right)$ for the tadpole improved case (70). With the parameters given above we get for the operators $(20)$

$$
B=\left(\begin{array}{cc}
-12.31636 & 0.16879 \\
0 & 13.29708
\end{array}\right)
$$

and

$$
B^{\mathrm{TI}}=\left(\begin{array}{cc}
-2.00613 & 0.16657 \\
0 & 4.72909
\end{array}\right) .
$$

Equations (74) and (75) show that the one-loop corrections on the diagonal have been reduced significantly. This is in accordance with the aims of tadpole improvement. 
The same procedure can be applied to the more complicated set $\mathcal{O}_{1}, \ldots, \mathcal{O}_{6}\left(\tau_{1}^{(8)}, C=-1\right)$ from (19). We obtain for the unimproved case the mixing matrix of renormalisation constants (lattice covariant derivative type $m=\mathrm{I}$ ) as

$$
\begin{aligned}
& Z^{(\mathrm{I})} \\
& =\left(\begin{array}{cccccc}
1.08602 & -0.01014 & -0.00259 & 0.00309 & 0.00011 & -0.00180 \\
0 & 0.79043 & 0 & 0 & 0 & 0 \\
-0.03386 & 0.07879 & 1.12164 & -0.05073 & 0.02642 & -0.02651 \\
0 & 0 & 0 & 0.79043 & 0 & 0 \\
0 & -0.02735 & 0 & 0 & 0.99751 & -0.01223 \\
0 & -0.02735 & 0 & 0 & -0.00408 & 0.98936
\end{array}\right) .
\end{aligned}
$$

The tadpole improved result reads

$$
\begin{aligned}
& Z^{\mathrm{TI},(\mathrm{I})} \\
& =\left(\begin{array}{cccccc}
1.15105 & -0.01637 & -0.00539 & 0.00487 & 0.00002 & -0.00272 \\
0 & 0.70666 & 0 & 0 & 0 & 0 \\
-0.05996 & 0.10966 & 1.20899 & -0.06762 & 0.03300 & -0.03328 \\
0 & 0 & 0 & 0.70666 & 0 & 0 \\
0 & -0.03667 & 0 & 0 & 0.99025 & -0.01223 \\
0 & -0.03667 & 0 & 0 & -0.00408 & 0.98210
\end{array}\right) .
\end{aligned}
$$

The renormalisation and mixing matrices for all other cases can be treated analogously. They show a similar behaviour: The $2 \times 2$ matrices exhibit rather small mixing coefficients, the other $6 \times 6$ problem $(21)$ is almost identical to the example discussed above.

\section{Summary}

In this paper we have considered quark-antiquark operators needed for the computation of the first two moments of GPDs and meson distribution amplitudes within the framework of lattice QCD. In one-loop lattice perturbation theory we have calculated the non-forward quark matrix elements of these operators employing clover improved Wilson fermions and Wilson's plaquette action for the gauge fields. From the results we have evaluated the matrices of renormalisation and mixing coefficients in the $\overline{\mathrm{MS}}$-scheme.

For the operators with only one derivative (relevant for the first moments) we could take over the numbers obtained for the first moments of ordinary parton distributions. The results for the second moments generalise the numbers calculated with Wilson fermions [14]. The general conclusions concerning the mixing properties remain unchanged.

If there is only mixing between one operator with two covariant derivatives $D$ and one operator with two external derivatives $\partial$, the mixing coefficient turns out to be rather small. In the two cases (19) and (21) with eight potentially mixing operators the mixing effects are more severe. Moreover, taking $\mathcal{O}_{1}$ from (19) or $\mathcal{O}_{1}^{5}$ from (21) as the operator to be measured in a numerical simulation, we find in each case mixing with a lower-dimensional operator (cf. (43) and (51)). This could lead to difficulties, because $1 / a$ effects are hard to get under control. For overlap fermions, however, these mixings with lower-dimensional operators of different chirality would be absent.

Additionally, we have discussed tadpole improvement with special attention to mixing operators. We have given the general prescription for mean field improvement and have shown how it works for selected examples.

Acknowledgements. This work is supported by DFG under contract FOR 465 (Forschergruppe Gitter-Hadronen-Phänomenologie) and by the EU Integrated Infrastructure Initiative Hadron Physics under contract number RII3-CT-2004-506078.

\section{References}

1. D. Müller, D. Robaschik, B. Geyer, F.M. Dittes, J. Hořejši, Fortschr. Phys. 42, 101 (1994) [hep-ph/9812448]

2. X.D. Ji, Phys. Rev. Lett. 78, 610 (1997) [hep-ph/9603249]

3. A.V. Radyushkin, Phys. Rev. D 56, 5524 (1997) [hep-ph/9704207]

4. M. Diehl, T. Gousset, B. Pire, J.P. Ralston, Phys. Lett. B 411, 193 (1997) [hep-ph/9706344]

5. J. Blümlein, B. Geyer, D. Robaschik, Nucl. Phys. B 560, 283 (1999) [hep-ph/9903520]

6. M. Diehl, Phys. Rep. 388, 41 (2003) [hep-ph/0307382]

7. V.L. Chernyak, A.R. Zhitnitsky, Phys. Rep. 112, 173 (1984)

8. V.M. Braun, G.P. Korchemsky, D. Müller, Prog. Part. Nucl. Phys. 51, 311 (2003) [hep-ph/0306057]

9. LHPC Collaboration, P. Hägler et al., Phys. Rev. D 68, 034505 (2003) [hep-lat/0304018]

10. QCDSF Collaboration, M. Göckeler et al., Phys. Rev. Lett. 92, 042002 (2004) [hep-ph/0304249]

11. QCDSF Collaboration, M. Göckeler et al., Nucl. Phys. Proc. Suppl. 140, 399 (2005) [hep-lat/0409162]

12. QCDSF Collaboration, M. Göckeler et al., Nucl. Phys. A 755, 537 (2005) [hep-lat/0501029]

13. QCDSF/UKQCD Collaboration, M. Göckeler et al., Phys. Lett. B 627, 113 (2005) [hep-lat/0507001]

14. M. Göckeler, R. Horsley, H. Perlt, P.E.L. Rakow, A. Schäfer, G. Schierholz, A. Schiller, Nucl. Phys. B 717, 304 (2005) [hep-lat/0410009]

15. B. Sheikholeslami, R. Wohlert, Nucl. Phys. B 259, 572 (1985)

16. M. Göckeler, R. Horsley, H. Perlt, P.E.L. Rakow, A. Schäfer, G. Schierholz, A. Schiller, Nucl. Phys. Proc. Suppl. 153, 269 (2006) [hep-lat/0511041]

17. QCDSF Collaboration, M. Göckeler et al., Phys. Rev. D 71, 114511 (2005) [hep-ph/0410187]

18. M. Göckeler, R. Horsley, E.-M. Ilgenfritz, H. Perlt, P. Rakow, G. Schierholz, A. Schiller, Phys. Rev. D 54, 5705 (1996) [hep-lat/9602029]

19. S. Capitani, M. Göckeler, R. Horsley, H. Perlt, P.E.L. Rakow, G. Schierholz, A. Schiller, Nucl. Phys. B 593, 183 (2001) [hep-lat/0007004]

20. H. Kawai, R. Nakayama, K. Seo, Nucl. Phys. B 189, 40 (1981)

21. G.P. Lepage, P.B. Mackenzie, Phys. Rev. D 48, 2250 (1993) [hep-lat/9209022] 